The Egyptian Journal of Hospital Medicine (January 2022) Vol. 86, Page 413-419

\title{
Osteoprotegerin, Soluble Receptor Activator Nuclear Factor-кB Ligand, Nuclear Factor Kappa $B$ and Intestinal Trefoil Factor 3 are Promising Biomarkers in Diagnosis and Follow- up Inflammatory Bowel Disease Patients
} Ragaa HM Salama ${ }^{1 *}$, Mohammed A Medhat ${ }^{2}$, Shimaa A Elghazally ${ }^{3}$, Nesma G Farag ${ }^{1}$, Aya A El Sanory ${ }^{4}$, Mohamed O Herdan ${ }^{5}$, Tasneem A El Sanory ${ }^{6}$, Amira A. Kamel ${ }^{1}$ ${ }^{1}$ Department of Medical Biochemistry, ${ }^{2}$ Department of Tropical Medicine, ${ }^{3}$ Department of Public Health, ${ }^{4}$ Department of Nuclear Medicine, South Egypt cancer Institute, ${ }^{5}$ Department of General Medicine, Faculty of Medicine, ${ }^{6}$ Department of Biochemistry, Faculty of Pharmacy, Assiut University, Assiut, Egypt

*Corresponding Author: Ragaa H M Salama, Tel: (+20)1063492008, P.O: 51717, E-mail: ragaa_2002@yahoo.com

\begin{abstract}
Background: Inflammatory bowel disease (IBD) is a condition in which the gastrointestinal system becomes inflamed for no apparent reason. Its incidence increased during the last few decades. As a result, it's critical to understand how to validate a clinical diagnosis and monitor any progress using a simple procedure. Osteoprotegerin (OPG), which interacts with RANKL, regulates RANK/RANKL signaling. OPG is a decoy receptor for soluble receptor activator of nuclear factor-B ligand (sRANKL), which stimulates nuclear factor kappa-light-chain-enhancer of activated B cells (NF- $\mathrm{KB}$ ) and the healing trefoil factor 3 (TFF3), which could be employed as in the diagnosis and follow-up of IBD patients, biomarkers are used. Objectives: The aim of the current work was to evaluate the role of RANKL (sRANKL), OPG, NF$\kappa \mathrm{B}$, and TFF3 in in the diagnosis of active IBD. Subjects and methods: Thirty-five newly diagnosed untreated IBD patients and thirty-five healthy controls were included in this study. Patients were then given particular treatment either in the form of cortisone, mesalamin, immunotherapy or infliximab and immunotherapy for 3 months and attend for second visit. OPG, sRANKL, NF- $\mathrm{kB}$ and TFF3 levels were estimated by ELISA method using commercial kits in patients before and after treatment and healthy control. Results: Patients with IBD had significantly increased levels of OPG, sRANKL, NF-B, and TFF3 than healthy controls $(\mathrm{P}=0.0001)$. After 3 months of treatment, patients' levels of NF-B and TFF3 were significantly lower $(\mathrm{p}=0.0001$ and 0.03 , respectively). Conclusion: The biomarkers OPG, sRANKL, NF- $\mathrm{BB}$, and TFF3 show promise in the diagnosis of active IBD. The decrease in NF- $\mathrm{KB}$ and TFF3 following treatment suggests that be used as biomarkers to track disease activity and treatment effectiveness.
\end{abstract}

Keywords: Inflammatory bowel disease, OPG , sRANKL, NFאB ,TFF3.

\section{INTRODUCTION}

IBDs are a set of chronic gastrointestinal inflammatory illnesses that affect millions of people around the world. The disease's progression is unpredictable, with flares complicating matters, as well as the necessity for long-term medication and, in some cases, surgery ${ }^{(1)}$. Although the cause of IBD is unknown, A dysregulated intestinal mucosal immune response, genetic factors, gut microbiota, and environmental factors are all thought to play a role in its development. ${ }^{(2,3)}$.

The most essential use of serological biomarkers is to assess disease aggressiveness, complications, or the need for surgery, as well as to predict illness prognosis ${ }^{(4)}$. However, have limited utility in establishing an initial definitive diagnosis for IBD. As a result, future research should focus on more effective biomarkers with high sensitivity and specificity ${ }^{(5)}$.

Osteoprotegerin is a protein that belongs to the tumour necrosis factor receptor superfamily and is involved in bone turnover, inflammatory pathways, and cell survival ${ }^{(6)}$. It acts as a decoy receptor for nuclear factor-B ligand soluble receptor activator (sRANKL), a chemotactic factor that enhances inflammatory responses in $\mathrm{T}$ cells by stimulating chemokine release, matrix metalloproteinase activity, and chemokine release ${ }^{(7)}$. OPG dimerization is required for RANK/RANKL inhibition because it bind with high affinity for RANKL. RANK/RANKL signalling inhibition may improve the efficacy of checkpoint blockade in cancer treatment. When treated as monotherapy, anti-RANKL monoclonal antibodies (mAbs) have limited antimetastatic activity ${ }^{\left({ }^{8}\right)}$. When sRANKL binds to its membrane receptor RANK, the receptor becomes trimerized, triggering TNF receptor-related components, adaptor proteins, and downstream signalling pathways, such as NF- $\kappa B$, are all recruited. ${ }^{(9)}$. Inflammatory stimuli activate NF- $\kappa \mathrm{B}$, a transcription factor that activates the inhibitor of kappa $B$ (IB) kinase in the cytosol.. Following that, NF- $\kappa B$ migrates to the nucleus via canonical or non-canonical signaling pathways, triggering the targeted genes that promote the development of pro-inflammatory cells including monocytes, macrophages, $\mathrm{T}$ and $\mathrm{B}$ cells ${ }^{(10)}$.

Intestinal trefoil factor 3 (TFF3) is largely generated and secreted by intestinal mucosa goblet cells ${ }^{(11)}$, which

This article is an open access article distributed under the terms and conditions of the Creative Commons Attribution (CC BY-SA) license (http://creativecommons.org/licenses/by/4.0/) 
is a hallmark of intestinal healing. To protect the intestinal mucosa, it normally works in tandem with mucins 2 which is the primary protein component of a mucus gel. (12)

The current study looked into a group of linked serological biomarkers, including OPG and sRANKL, as well as the involvement of NF- $\kappa \mathrm{B}$ in activating this pathway. TFF3 was also used to assess mucosal healing in adult newly diagnosed, untreated patients with active IBD, as well as their serum level after three months of particular treatment.

\section{PATIENTS AND METHODS}

This case-control study included a total of thirty-five newly diagnosed untreated IBD patients and thirty-five healthy controls, attending at outpatient clinic, Department of Tropical Medicine and Gastroenterology, Al-Rajhy Liver Hospital, Assiut University.

\section{Ethical Consideration:}

The study was authorized by the Ethical Committee, Faculty of Medicine, Assiut University (IRB no:17100659), and each participant signed an informed written consent form. This research was carried out in conformity with the World Medical Association's Code of Ethics (Declaration of Helsinki) for human studies. The laboratory analysis was performed at Assiut University's Medical Research Centre, Faculty of Medicine.

All patients were evaluated clinically, endoscopically, and histopathologically. The Guidelines developed by the European Crohn's and Colitis Organization (ECCO) and the European Society of Gastrointestinal and Abdominal Radiology (ESGAR) ${ }^{(13)}$ were used to diagnose the patients. They had no signs of autoimmune disease, cardiovascular disease, or diabetes. The healthy control group included volunteers of similar age and sex who showed no indications of IBD or other immune-mediated illnesses and no family history of IBD or other immune-mediated disorders.

The first sample was obtained at baseline, after endoscopic diagnosis (before treatment was initiated). Thereafter, patients received specific treatment in the form of cortisone, mesalamine, immunotherapy or infliximab and immunotherapy according to the step-up approach, as recommended by the Dutch guidelines after excluding patients with positive tuberculin test, positive hepatitis B surface antigen and patients with HIV infection. Then, the second sample was collected at a second visit to the hospital, with an interval of at least three months from the first one.

The 35 active IBD patients were 25 with UC and 10 with $\mathrm{CD}$ and twenty-two of those patients were committed with the second visit 3 months after treatment. Twelve of them were on cortisone, mesalamine and immunotherapy. On the other hand, 10 were on infliximab and immunotherapy. All patients and healthy controls were subjected to measurement of OPG, sRANKL, NF$\mathrm{B}$, and TFF3 by ELISA technique. The tests were carried out according to the manufacturer's protocol, which was provided by Glory Science Co., Ltd, China; catalogue numbers 16172, 11022, 10947, and 17165, respectively.

\section{Statistical analysis}

SPSS version 22 was used for data entry and analysis (Statistical Package for Social Science). The data was presented in the form of a number, a percentage, a mean, and a standard deviation. To compare qualitative variables, the Chi-square test was performed. To compare two quantitative variables, the Mann-Whitney test was utilised. To compare quantitative factors between patients with active IBD and patients 3 months after therapy, the Wilcoxon Signed Rank Test was used. To discover correlation between quantitative variables, Spearman correlation was used. The sensitivity, specificity, positive and negative predictive values, and ROC curves were calculated using the Medcalc tool. When P 0.05, the Pvalue is considered statistically significant.

\section{RESULT \\ Demographic, and clinical data:}

This study included 35 IBD patients (10 males and 25 females) and 35 healthy controls (12 males and 23 females). In terms of age and sex, there was no significant difference between IBD patients and controls. The level of hemoglobin in patients with active disease was substantially lower than in controls $(\mathrm{p}=0.0001)$. Patients with active disease had significantly greater ESR and CRP than controls $(\mathrm{p}=0.0001$ ).

The level of OPG, sRANKL, NF- $\kappa \mathrm{B}$ and TFF3 in active IBD patients (Group I), patients 3 months after treatment (Group II) and healthy control (Group III) are presented in (Table 1). During the active stage of IBD, OPG $(\mathrm{P}=0.0001), \quad$ sRANKL $\quad(\mathrm{P}=0.0001), \quad \mathrm{NFB} \quad(\mathrm{P}$ $=0.0001)$, and TFF3 $(\mathrm{P}=0.0001)$ were substantially higher in IBD patients than in controls.

After 3 months of treatment, patients' serum levels of OPG and sRANKL got reduced when compared to the same patients in the active stage, although the difference was not significant $(\mathrm{P}=0.7$ and 0.4 , respectively). However, serum levels of NF-B and TFF3 were considerably lower in IBD patients after 3 months of treatment $(\mathrm{p}=0.0001$ and 0.03 , respectively) when compared to the same patients during the active period. After 3 months of treatment, serum levels of OPG $(\mathrm{P}=0.0001)$, sRANKL $(\mathrm{P}=0.002)$, and TFF3 $(\mathrm{P}=0.0001)$ were considerably higher in IBD patients than in controls. After 3 months of treatment, serum levels of NF-B ( $\mathrm{P}=$ $0.06)$ reveal no significant difference in IBD patients compared to controls $(\mathrm{P}=0.06)$. 
Table (1): Serum levels of OPG, sRANKL, NF-KB and TFF3 in inflammatory bowel disease patients during active stage (Group I), 3 months after treatment (Group II) and healthy control (Group III).

\begin{tabular}{|c|c|c|c|c|}
\hline Markers & $\begin{array}{c}\text { group I } \\
(\mathrm{n}=22) \\
\text { Active stage }\end{array}$ & $\begin{array}{c}\text { group II } \\
(\mathrm{n}=22) \\
\text { After treatment }\end{array}$ & $\begin{array}{c}\text { group III } \\
(n=35) \\
\text { Healthy control }\end{array}$ & P-value \\
\hline \multicolumn{5}{|l|}{ OPG (ng/ml) } \\
\hline Mean \pm SD & $1.00 \pm 0.25$ & $0.97 \pm 0.22$ & $0.68 \pm 0.19$ & $\begin{array}{l}\mathrm{P} 1=0.7 \\
\mathrm{P} 2=0.0001 \\
\mathrm{P} 3=0.0001\end{array}$ \\
\hline Median & 0.96 & 0.95 & 0.56 & \\
\hline \multicolumn{5}{|c|}{ sRANKL(pg/ml) } \\
\hline Mean \pm SD & $19.01 \pm 3.62$ & $17.69 \pm 5.86$ & $13.83 \pm 2.12$ & $\begin{array}{l}\mathrm{P} 1=0.4 \\
\mathrm{P} 2=0.002 \\
\mathrm{P} 3=0.0001\end{array}$ \\
\hline Median & 19.88 & 18.2 & 13.2 & \\
\hline \multicolumn{5}{|l|}{ NF-кB (pg/ml) } \\
\hline Mean \pm SD & $649.57 \pm 46.58$ & $107.11 \pm 5.63$ & $105.81 \pm 24.12$ & $\begin{array}{l}\mathrm{P} 1=0.0001 \\
\mathrm{P} 2=0.06 \\
\mathrm{P} 3=0.0001\end{array}$ \\
\hline Median & 632.5 & 98.1 & 99.1 & \\
\hline \multicolumn{5}{|l|}{ TFF3 (pg/ml) } \\
\hline Mean \pm SD & $77.54 \pm 13.54$ & $57.08 \pm 11.74$ & $55.82 \pm 13.87$ & $\begin{array}{l}\mathrm{P} 1=0.03 \\
\mathrm{P} 2=0.0001 \\
\mathrm{P} 3=0.0001\end{array}$ \\
\hline Median & 69.3 & 67.7 & 56.4 & \\
\hline
\end{tabular}

P1:

A paired T test was used to compare Group I and Group II.

P2: The unpaired T test was used to compare Group II and Group III.

P3: An unpaired T test was used to compare Group I with Group III.

A statistically significant $P$ value is less than 0.05 .

$0.82>0.82 \mathrm{ng} / \mathrm{ml}$ was the best OPG cutoff value for detecting IBD illness, with a sensitivity of 77.1 percent and specificity of 74.2 percent (area under the ROC was 0.792). While the cutoff value for sRANKL level was > $13.73 \mathrm{pg} / \mathrm{ml}$ with $94.2 \%$ sensitivity and $68.6 \%$ specificity (area under the curve was 0.892). For NF- $\kappa B$ the cutoff value was > $175.13 \mathrm{pg} / \mathrm{ml}$ with $94.3 \%$ sensitivity and $97.1 \%$ specificity for diagnosis of IBD (area under the curve 0.998). The cutoff value for serum TFF3 level was $>61.09 \mathrm{pg} / \mathrm{ml}$ with $77.1 \%$ sensitivity and $74.3 \%$ specificity (area under the curve 0.736 ) to detect healing of intestinal mucosa and remission state as shown in Figure 1. 


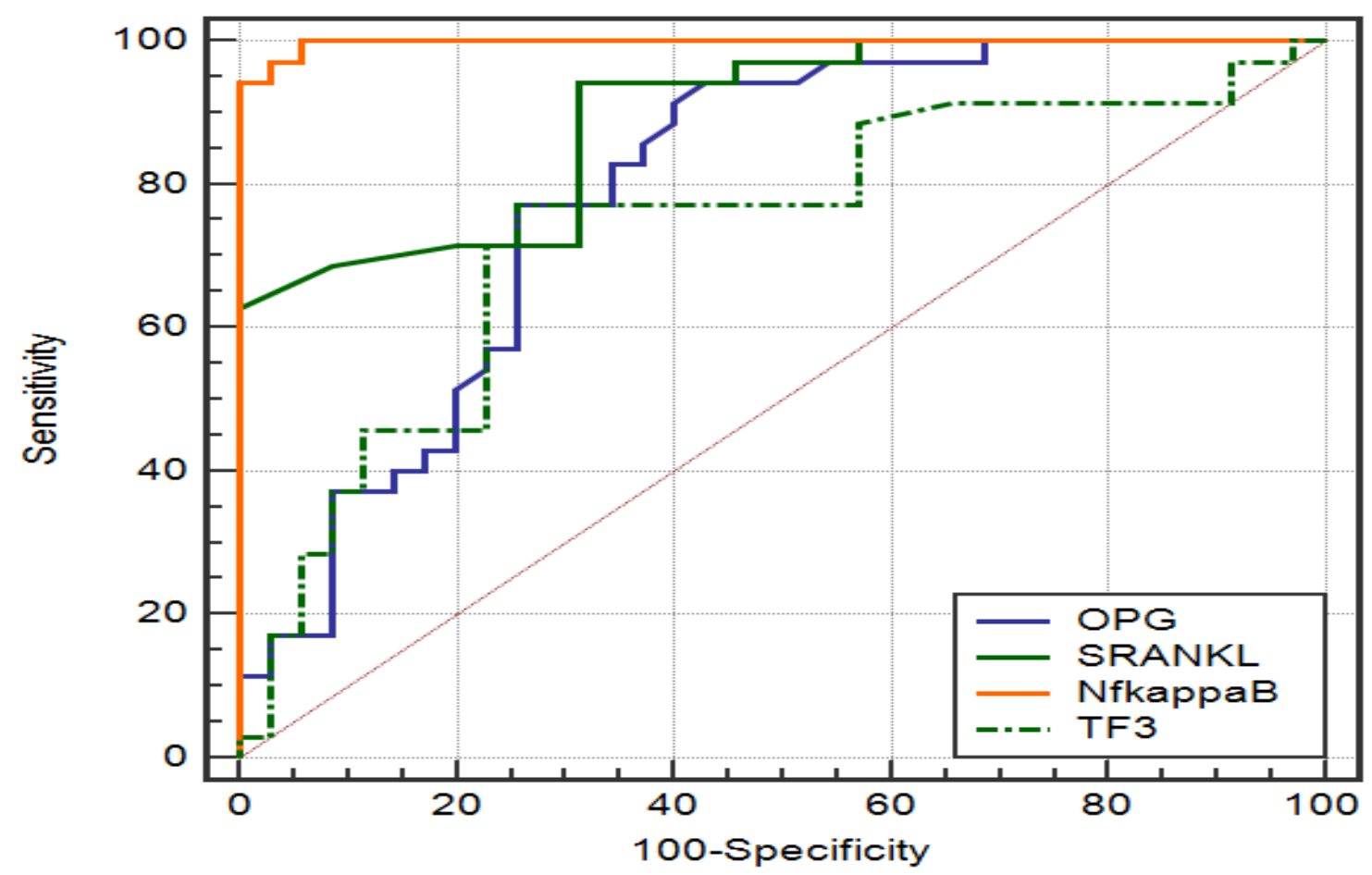

Fig. 1: Curve of receiver operating characteristic (ROC) of OPG, sRANK1, NF- $\kappa B$ and TFF3.

There was no significant change in serum levels of OPG, sRANKL, NF- $\kappa B$, and TFF3 in IBD patients after 3 months of treatment who got cortisone, mesalamin, and immunotherapy versus those who received immunotherapy plus infliximab $(\mathrm{p}>0.05)($ Table 2).

Table (2): Patients with ulcerative colitis and crohn's disease serum levels of OPG, sRANKL, NF- $\mathrm{kB}$, and TFF3.

\begin{tabular}{|c|c|c|c|}
\hline \multirow{2}{*}{ Markers } & \multicolumn{2}{|l|}{ Diagnosis } & \multirow{2}{*}{ P-value } \\
\hline & Ulcerative colitis & Crohn's disease & \\
\hline \multicolumn{4}{|l|}{ OPG (ng/ml) } \\
\hline Mean \pm SD & $1.06 \pm 0.29$ & $1.05 \pm 0.20$ & 0.2 \\
\hline Median & 0.97 & 0.93 & \\
\hline \multicolumn{4}{|c|}{ sRANKL (pg/ml) } \\
\hline Mean \pm SD & $18.09 \pm 3.47$ & $22.49 \pm 2.16$ & 0.1 \\
\hline Median & 18.02 & 22.72 & \\
\hline \multicolumn{4}{|l|}{ NF-kB (pg/ml) } \\
\hline Mean \pm SD & $676.29 \pm 80.50$ & $762.03 \pm 54.75$ & 0.3 \\
\hline Median & 682.6 & 653.2 & \\
\hline \multicolumn{4}{|l|}{ TFF3 (pg/ml) } \\
\hline Mean \pm SD & $83.46 \pm 14.74$ & $71.19 \pm 11.59$ & 0.1 \\
\hline Median & 70.5 & 71.2 & \\
\hline
\end{tabular}

The correlation coefficient(r) of the studied parameters in patient in active disease showed significant positive correlations of OPG. NF- $\kappa \mathrm{B}$ was strongly positively linked with ESR first hour $(\mathrm{r}=0.5, \mathrm{P}=.0001)$, ESR second hour ( $\mathrm{r}=0.6$, $\mathrm{P}=0.0001)$, and $\mathrm{CRP}(\mathrm{r}=0.4, \mathrm{P}=0.003)$ with age $(\mathrm{r}=0.4, \mathrm{P}=0.01)$, indicating that it correlates with disease activity.

NF- $\kappa B$ in UC patients was also significantly positively connected with ESR first hour ( $\mathrm{r}=0.5, \mathrm{P}=0.003)$, ESR second hour ( $\mathrm{r}=0.7, \mathrm{P}=0.0001)$, and $\mathrm{CRP}(\mathrm{r}=0.4, \mathrm{P}=0.03)$, according to the correlation coefficient ( $\mathrm{r}$ ) of the examined parameters in UC patients and CD. In CD patients, NF- $\kappa \mathrm{B}$ was shown to be substantially linked with $\mathrm{ESR}$ first hour ( $\mathrm{r}=0.6, \mathrm{P}=0.03$ ).. 


\section{DISCUSSION}

The current gold standard for detecting and monitoring disease activity in IBD patients is an endoscopic examination, but it is overly complicated, time-consuming, expensive and intrusive. Blood biomarkers have the potential to revolutionize the way IBD is diagnosed and treated.

Female patients make up the bulk of the participants in this study, as opposed to males. It was in line with the findings of Conley et al ${ }^{(14)}$. Because alterations in intestinal permeability and immune function are expected to have a part in the genesis of IBD, estrogen is thought to play a role ${ }^{(15)}$.

Because anemia is common in IBD patients as a result of deficiencies in iron, B12, and folic acid, bleeding from mucosal lesions, surgery, systemic inflammation, and drugs are all factors to consider ${ }^{(16)}$, the current study's findings revealed that haemoglobin levels in IBD patients were substantially reduced in comparison to the controls. Rempel et al results ${ }^{(17)}$ investigation support this ESR (first and second hour) and CRP were similarly considerably higher in active IBD patients than in controls, according to our research. Teng et al. ${ }^{(18)}$ and Sowiska-Solnica et al. (19) both came to similar conclusions.

Inflammatory pathways are affected by interactions between OPG, sRANKL, and RANK. When RANKL binds to RANK, the receptor becomes trimerized, NF- $\kappa \mathrm{B}$ is activated by stimulating the recruitment of TNF receptor associated components, adaptor proteins, and downstream signaling pathways. $\mathrm{NF}-\mathrm{\kappa B}$ expression in mucosal macrophages increases when the NF- $\kappa B$ pathway is activated. The current investigation found that serum levels of OPG were considerably greater in active IBD patients than in controls $(\mathrm{p}=0.0001)$. TNF, interleukin-6 (IL-6) and interleukin-1B (IL-1B) are proinflammatory cytokines that induce inflammation and expression of the OPG gene in smooth muscle cells and endothelium as a result of the regulation of the inflammatory process, according to our findings ${ }^{(21)}$. Because proinflammatory factors have been identified as a major element in the development of IBD, OPG and their concentration play a function in the progression of IBD ${ }^{(22)}$. Our findings are consistent with a Krela-Kamierczak et al. ${ }^{(23)}$ investigation, which supports this interpretation.

The current study found that patients' serum levels of OPG dropped after 3 months of treatment when compared to the same patients in the active period, although The change was not statistically significant ( $>0.05$ ). It might be argued that reaching the fundamental level of OPG takes a longer duration of therapy and make a noticeable difference between the two groups (24). In addition, after three months of therapy, there was a significant difference in OPG between patients and controls $(\mathrm{P}<0.05)$. It could be explained by the fact that not all improvement means the patient is in remission, Clinical remission was defined as the elimination of clinical symptoms after induction treatment at a general physician's assessment, in combination with endoscopic remission, normalisation of radiological examination, and/or a decrease in inflammatory markers such as CRP or faecal calprotectin (4).

In our investigation, serum OPG levels in IBD patients were found to be significantly related to age. This is consistent with Stanisawowski, M., et al findings's ${ }^{25}$, proposed a hypothesis that OPG may play a role in IBD patients as they age, and that a greater OPG serum level was linked to increased bone resorption ${ }^{(25)}$.

The current study found that sRANKL levels in the blood were considerably greater in active IBD patients than in controls. $(p=0.0001)$. The interconnections of OPG, sRANKL, and RANK are important in inflammatory pathways, and RANKL-RANK binding activates pathways that help T-lymphocytes and dendritic cells survive ${ }^{26}$. Krela-Kamierczak et al. ${ }^{(23)}$, There was no substantial difference between patients and controls, on the other hand, which contradicts our findings.

The current study also found that after 3 months of treatment, Patients' blood levels of sRANKL decreased when compared to the same patients during the active period, but the difference was not statistically significant ( $>$ > 0.05). As sRANKL may require a longer treatment period to reach the basic level and make a meaningful difference between the two groups ${ }^{24}$. These findings are similar to those of Galliera et al. ${ }^{(24)}$.

Our findings revealed that NF- $\mathrm{BB}$ levels in active IBD patients were considerably greater than matched in age and sex, healthy peers $(\mathrm{p}=0.0001)$. Increased NF- $\mathrm{KB}$ expression in mucosal macrophages, pro-inflammatory cytokines are produced as a result such TNF, IL1, and IL6, which damage mucosal tissue 20. In colonic epithelial cells, increased expression of intercellular adhesion molecule 1 (ICAM1), which aids in the recruitment of neutrophil granulocytes to the site of inflammation, is also connected to NF- $\kappa \mathrm{B}$.. NF$\kappa \mathrm{B}$ produces cytokines that stimulate, activate, and develop immune cells in the lamina propria, aggravating the persistence of mucosal inflammation ${ }^{(20)}$.

$\mathrm{NF}-\kappa \mathrm{B}$ expression was significantly higher in IBD patients compared to controls, according to Hegazy et al. ${ }^{27}$, which supports our hypothesis. Han et al. ${ }^{28}$ conducted a study on $\mathrm{CD}$ patients and found that they had a high level of NF- $\kappa \mathrm{B}$ activation.

In our study, we observed a significant decline in the NF- $\kappa \mathrm{B}$ in IBD patients after 3 months of therapy compared to those in active stage $(\mathrm{p}=0.0001)$. We predicted that glucocorticoids and antiTNF interact with $\mathrm{NF}-\kappa \mathrm{B}$, resulting in a decrease in pro-inflammatory 
cytokines, adhesion molecules, and nitric oxide synthesis, because IKB is increased, which lowers transcription of pro-inflammatory cytokines ${ }^{(29)}$.

In individuals with $\mathrm{UC}, \mathrm{NF}-\kappa \mathrm{B}$ was found to be significantly positively related with ESR first hour $(\mathrm{P}=0.003)$, ESR second hour $(\mathrm{P}=0.0001)$, and CRP $(\mathrm{P}=0.033)$. NF- $\kappa \mathrm{B}$ was shown to be significantly positively linked with ESR first hour in CD patients $(\mathrm{P}=0.038)$. Increased CRP activity is frequently related with NF- $\kappa \mathrm{B}$ activation ${ }^{(30)}$, according to the findings. The current investigation found that TFF3 levels in the blood were considerably greater in active IBD patients than in controls $(p=0.0001)$. Because TFF3 is secreted by intestinal goblet cells and is ordinarily seen in very minute amounts in the blood, the elevated levels of TFF3 in IBD patients' blood suggested that it was mostly derived from the damaged intestinal mucosa ${ }^{(18)}$.

Nakov et al. ${ }^{(31)}$ showed significantly greater levels of TFF3 in patients than in healthy controls, which agrees with our findings.

When compared to patients in the active stage, TFF3 levels in IBD patients fell dramatically after 3 months of treatment $(\mathrm{p}=0.03)$. TFF3 peptides are thought to interact with mucin polysaccharide chains, causing mucins to cross-link, increasing mucosal gel viscosity and protecting the underlying epithelium ${ }^{(32)}$, so these findings could be explained by healing of the intestinal mucosa being accompanied by a decrease in TFF3 levels. Teng et al. ${ }^{18}$ conducted a cohort analysis that agreed with our findings revealed that TFF3 was shown to be considerably higher in patients with active IBD than in those who were in remission $(\mathrm{p}<0.01)$.

Nakov et al. ${ }^{(31)}$ found that TFF3 levels in the blood were significantly higher in individuals with active IBD than in those who were in remission (p 0.001), which is consistent with our findings.

In the current study, TFF3 was found to be significantly positively related with CRP $(\mathrm{P}=0.032)$ in people with CD. Teng et al. ${ }^{18}$ reported a high link between TFF3 and CRP, and our data support their findings ( $\mathrm{P}$ $0.01)$.

There were no significant differences in any of the four biomarkers between patients with UC and those with CD. This could be explained by the fact that UC and $\mathrm{CD}$ have similar etiologies, with both including intestinal inflammation and damage, as well as the release of proinflammatory cytokines ${ }^{(33)}$. As a result, they can't tell the difference between the two.

Moschen et al., ${ }^{(34)}$ observed no significant differences between patients with UC and patients with $\mathrm{CD}$, and Teng et al.,18 reported no significant differences between UC and CD patients, which agrees with our findings. The levels of TFF3 in patients with UC and those with $\mathrm{CD}$ were not significantly different.

\section{CONCLUSION}

It could be concluded that OPG is the decoy receptor of sRANKL which bind with its receptor RANK and activate NF- $\mathrm{B}$. This pathway may involve in the pathogenesis of IBD and any interference with pathway may affect the progress of the disease.

Inhibition of RANK/RANKL as an immunomodulatory agent. While TFF3 is a good marker indicating healing of mucosa and a state of remission.

\section{ACKNOWLEDGMENT}

The Assuit Medical School Grants Office is gratefully acknowledged.

\section{Funding:}

This research received grant from Assuit Medical School Grant Office, grant number 2019-03-25-004-R2.

\section{Conflict of interest:}

All authors contributed to the critical assessment, editing, revision, and approval of the final article, and they state that they have no competing interests.

\section{REFERENCES}

1. Huang H, Fang M, Jostins L, Mirkov M, Boucher G, Anderson C et al. (2017): Fine-mapping inflammatory bowel disease loci to single-variant resolution, 547(7662):173-8.

2. Ikhtaire S, Shajib M, Reinisch W, Khan W ( 2016): Fecal calprotectin its scope and utility in the management of inflammatory bowel disease. J Gastroenterol., 51(5):434-46.

3. Bonneau J, Dumestre-Perard C, Rinaudo-Gaujous M et al.(2015): Systematic review: new serological markers (anti-glycan, anti-GP2, anti-GM-CSF Ab) in the prediction of IBD patient outcomes. Autoimmun Rev., 14(3):231-45.

4. Smids C, Horjus Talabur Horje C, Groenen M et al. (2017): The value of serum antibodies in differentiating inflammatory bowel disease, predicting disease activity and disease course in the newly diagnosed patient. Scandinavian journal of gastroenterology, 52(10):1104-12.

5. Zhou G, Song Y, Yang W et al. (2016): ASCA, ANCA, ALCA and Many More. Are They Useful in the Diagnosis of Inflammatory Bowel Disease? Digestive diseases (Basel, Switzerland), 34(1-2):90-7.

6. De Voogd F, Gearry R, Mulder C, Day A (2016): Osteoprotegerin: A novel biomarker for inflammatory bowel disease and gastrointestinal carcinoma. J Gastroenterol Hepatol., 31(8):1386-92.

7. Raaz-Schrauder D, Schrauder M, Stumpf C et al. (2017): Plasma levels of sRANKL and OPG are associated with atherogenic cytokines in patients with intermediate cardiovascular risk Heart Vessels, 32(11):1304-13.

8. Van Dam P, Verhoeven $Y$, Trinh $X$ et al. (2019): RANK/RANKL signaling inhibition may improve the effectiveness of checkpoint blockade in cancer treatment. Critical reviews in oncology/hematology, 133:85-91. 
9. González-Suárez E, Sanz-Moreno A (2016): RANK as a therapeutic target in cancer. The FEBS journal, 283(11):1833.

10. Choy K, Murugan D, Leong X, Abas R, Alias A, Mustafa M (2019):Flavonoids as Natural Anti-Inflammatory Agents Targeting Nuclear Factor-Kappa B (NFkappaB) Signaling in Cardiovascular Diseases: A Mini Review. Front Pharmacol., 10:1295.

11. Yoon H, Kim N (2015): Diagnosis and management of high risk group for gastric cancer. Gut and Liver,9(1):5-17.

12. Wang $Y$, Liang K, Kong W (2019): Intestinal Trefoil Factor 3 Alleviates the Intestinal Barrier Function Through Reducing the Expression of TLR4 in Rats with Nonalcoholic Steatohepatitis. Arch Med Res., 50(1):2-9.

13. Maaser C, Sturm A, Vavricka $S$ et al. (2019): ECCOESGAR Guideline for Diagnostic Assessment in IBD Part 1: Initial diagnosis, monitoring of known IBD, detection of complications. Journal of Crohn's and Colitis, 13(2):144$64 \mathrm{~K}$.

14. Conley S, Proctor D, Jeon S, Sandler R, Redeker N (2017): Symptom clusters in adults with inflammatory bowel disease. Research in nursing \& health, 40(5):424-34.

15. Lophaven S, Lynge E, Burisch J( 2017): The incidence of inflammatory bowel disease in Denmark 1980-2013: a nationwide cohort study. Alimentary pharmacology \& therapeutics, 45(7):961-72.

16. Dignass A, Gasche C, Bettenworth D et al.( 2015): European consensus on the diagnosis and management of iron deficiency and anaemia in inflammatory bowel diseases. Journal of Crohn's and Colitis, 9(3):211-22.

17. Rempel J, Grover K, El-Matary W( 2021): Micronutrient Deficiencies and Anemia in Children with Inflammatory Bowel Disease. Nutrients, 13, 236.

18. Teng X, Yang Y, Liu L et al. (2020): Evaluation of inflammatory bowel disease activity in children using serum trefoil factor peptide. Pediatric research, 88(5):792-5.

19. Słowińska-Solnica K, Pawlica-Gosiewska D, Gawlik K, Owczarek D, Cibor D, Pocztar H et al. (2021): Serum inflammatory markers in the diagnosis and assessment of Crohn's disease activity. Archives of Medical Science, 17(1):252.

20. Lu D, Zhao Y (2020): Targeting NF- $\mathrm{B}$ p pathway for treating ulcerative colitis: comprehensive regulatory characteristics of Chinese medicines. Chinese medicine, 15(1): 15 .

21. Bandarra D, Rocha $S$ (2015): HIF-1 $\alpha$, a novel piece in the NF- $\kappa B$ puzzle. Infammation and Cell Signaling, 2(1):e792.

22. Van Dam P, Verhoeven Y, Jacobs J et al. (2019): RANKRANKL signaling in cancer of the uterine cervix: a review. International journal of molecular sciences, 20(9):2183.

23. Krela-Kaźmierczak I, Szymczak-Tomczak A, Lykowska-Szuber L et al. (2018): Interleukin 6, osteoprotegerin, sRANKL and bone metabolism in inflammatory bowel diseases. Advances in Clinical and Experimental Medicine, 27(4):449-53.

24. Galliera E, De Girolamo L, Dogliotti G, De Salvo C, Tosetti G, Pastorelli L (2011): Circulating OPG levels are reduced following infliximab treatment and correlate with CRP levels: is serum OPG a potential marker of IBD disease activity? Inflammatory bowel diseases, 17(6):E59-E60.

25. Stanisławowski M, Wiśniewski P, Guzek M et al. (2014): Influence of receptor activator of nuclear factor kappa B ligand, osteoprotegerin and interleukin-33 on bone metabolism in patients with long-standing ulcerative colitis. Journal of Crohn's and Colitis, 8(8):802-10.

26. De Voogd F, Gearry R, Mulder C, Day A (2016): Osteoprotegerin: A novel biomarker for inflammatory bowel disease and gastrointestinal carcinoma. Journal of gastroenterology and hepatology, 31(8):1386-92.

27. Hegazy S, El-Bedewy M (2010): Effect of probiotics on pro-inflammatory cytokines and $\mathrm{NF}-\mathrm{\kappa B}$ activation in ulcerative colitis. World journal of gastroenterology, 16(33):4145.

28. Han Y, Koh J, Kim J(2017): NF-kappa B activation correlates with disease phenotype in Crohn's disease, 12(7): 0182071.

29. Szatkowski P, Krzysciak W, Mach T, Owczarek D, Brzozowski B, Szczeklik K. (2020): Nuclear factor- $\kappa B$ importance, induction of inflammation, and effects of pharmacological modulators in Crohn's disease. Journal of physiology and pharmacology : an official journal of the Polish Physiological Society, 71(4):453-465.

30. Sheng F, Cheng L, Zeng Q, Gao W (2009): Increased expression and activity of MMP-9 in C-reactive proteininduced human THP-1 mononuclear cells is related to activation of nuclear factor kappa-B. Journal of Huazhong University of Science and Technology Medical sciences, 29(4):399-403.

31. Nakov R, Velikova T, Nakov V, Ianiro G, Gerova V, Tankova L (2019): Serum trefoil factor 3 predicts disease activity in patients with ulcerative colitis. Eur Rev Med Pharmacol Sci., 23(2):788-94.

32. Thim L, Madsen F, Poulsen S (2002): Effect of trefoil factors on the viscoelastic properties of mucus gels. European journal of clinical investigation, 32(7):519-27.

33. McGovern D, Kugathasan S, Cho J (2015): Genetics of Inflammatory Bowel Diseases. Gastroenterology, 149(5):1163-76 e2.

34. Moschen A, Kaser A, Enrich B et al. (2005): The RANKL/OPG system is activated in inflammatory bowel disease and relates to the state of bone loss. Gut, 54(4):47987. 